\title{
Parametric study of corn cob biochar (CCBc) yield via microwave pyrolysis
}

\author{
Kristine Iannah Lawas, Stephen Doliente, Rowena Carpio*, Veronica Migo, and Catalino Alfafara \\ ${ }^{1}$ Department of Chemical Engineering, College of Engineering and Agro-Industrial Technology, University of the \\ Philippines Los Banos, 4030 Laguna, Philippines
}

\begin{abstract}
In the long-run, microwave pyrolysis can be a simpler and low energy-requiring alternative to conventional pyrolysis for the thermochemical conversion of biomass to useful products. However, there are still research gaps in its mechanism. Thus, this study investigated the various factors affecting the biochar yield using a half resolution $\left(2^{\mathrm{k}-1}\right)$ factorial design on the microwave pyrolysis of corn cob wastes. A viable biochar product was produced within minutes of the reaction; wherein, the statistical analysis confirmed the exposure time, microwave output power and their interaction as significant in the $\mathrm{CCBc}$ yield. The highest yield obtained was $52.87 \%$ when exposure time and output power were set to $5 \mathrm{~min}$ and $450 \mathrm{~W}$, respectively. A general decreasing effect on the yield was observed from increasing exposure time and output power. This was due to the rapid heating experienced by the corn cob wastes causing the hydrocarbons to react and transform into permanent gases at higher temperatures. To confirm the carbon content of the $\mathrm{CCBc}$, elemental analysis showed an average of $67.11 \% \mathrm{C}$ at low time-low power (LTLP) of $450 \mathrm{~W}$ for $5 \mathrm{~min}$ and $81.32 \% \mathrm{C}$ for the samples operated at high time- high power (HTHP) of $700 \mathrm{~W}$ for $10 \mathrm{~min}$.
\end{abstract}

\section{Introduction}

Biomass is foreseen to have a much greater significant role in humanity's carbon-free future supply of materials, fuel and energy. In the Philippines, renewable sources like biomass can be easily sourced from agricultural crops and their residues. Locally, there are over 30 million hectares of arable land with about $47 \%$ dedicated to growing crops [1]. Corn, being one of the top agricultural products in the country, is generated at 4.96 million tons per year [1] However, high throughput of corn consequently creates high amounts of waste in the form of corn cobs and corn stover which are underutilized in the country.

In lieu of decarbonizing economies towards sustainability, pyrolysis is a well-known waste valorization technology which converts biomass wastes into useful fuels, chemicals and materials. It produces biogas for fuel [2], bio-oil for asphalt applications and recently biochar for gas separation [3], catalyst application [4], carbon sequestration [5] and even electrode nanomaterials [6].

Traditionally, pyrolysis is conducted in pressurized and complex reactor vessels with significant input of heat at prolonged amounts of time. Moreover, pre-processing requires the drying of biomass to increase the calorific value of the feedstock [7]. To circumvent the complexity and input of additional energy, a growing body of research shows microwave processing to be a beneficial alternative $[4,8,9]$. Ingole et. al [10] listed various factors affecting microwave assisted pyrolysis namely biomass type and size, moisture content, reaction temperature and time, microwave output power and type, reactor design, microwave absorber, catalyst, agitation and carrier gas. In this study, microwave output power, reaction time and absorber loading were investigated since there were no studies integrating such factors and these were individually identified as key players in controlling biochar yield as published in various studies [11][12] [13].

A study by Wang et. al [14] investigated the transformation of biomass into carbon by using a domestic microwave as pyrolysis reactor with the aid of a microwave absorber. It also follows an outward heating profile contrary to the inward profile of common heating mechanisms [15]. This creates a more uniform heat transfer within the sample which makes it a potential method for pyrolysis. Reaction time greatly decreased from hours in conventional methods to minutes inside a microwave which also lowered operational cost. However, there are little to no studies about the effects and interaction of various operating parameters such as exposure time, power, and amount of microwave absorber in production of biochar, especially in corn cob wastes. To trail braze the process development of microwave pyrolysis, the position of this study was to determine the significant factors through a parametric analysis affecting the production of corn cob biochar $(\mathrm{CCBc})$.

* Corresponding author: kalawas@up.edu.ph 


\section{Material and methods}

\subsection{Materials and reagents}

A Samsung Me711K domestic microwave oven was used for the reactor. Moreover, a 50-cc quartz boiling flask lined with Teflon tape was fitted to a condenser to form a reactor vessel. Corn cobs were size reduced and sieved using a mesh 4 screen. Pyrrole monomer and $\mathrm{FeCl} 3$ hexahydrate was used for the polymerization of polypyrrole.

\subsection{Experimental set-up}

This study investigated significant factors affecting biochar yield by using a half- resolution $2^{\mathrm{k}-1}$ factorial. The five factors investigated in the study are namely: (1) microwave exposure time, (2) catalyst concentration, (3) pyrrole concentration, (4) polymerization time of polypyrrole, and (5) microwave output power.

Figure 1 shows the experimental setup used in the pyrolysis of corn cob wastes. Five grams of the biomass was placed in an Erlenmeyer flask which was initially added with $50 \mathrm{~mL}$ of the pyrrole solution. It was let to stand for at least 2 minutes for proper wetting. Next, another $50 \mathrm{~mL}$ of the hexahydrate solution was added into the mixture. The in-situ formation of polypyrrole was denoted by the darkening of the solution. Next, the flasks were covered with aluminum foil to prevent contact with the environment.

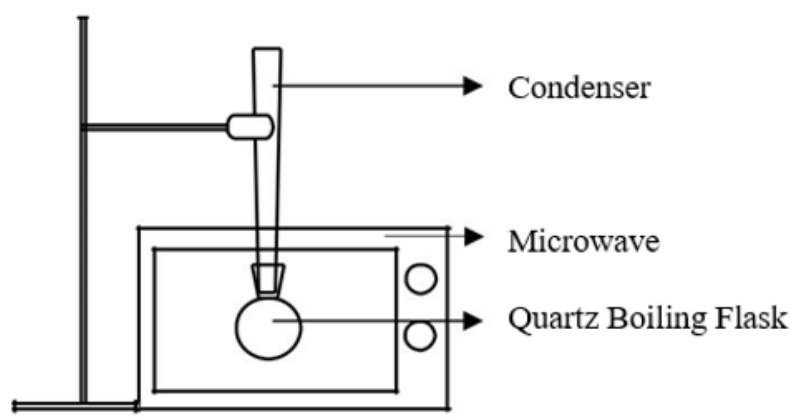

Figure 1. Experimental setup for pyrolysis experiment

After 24 hours of in-situ polymerization under ambient conditions, the mixtures were filtered and pyrolyzed at varying time (5 min and $10 \mathrm{~min}$ ) and output power ( $450 \mathrm{~W}$ and $700 \mathrm{~W}$ ). Table 1 show the combination of factors used in the experiment.

Table 1. Factors and levels used in biochar production.

\begin{tabular}{cccccc}
\hline $\begin{array}{c}\text { Leve } \\
\text { l }\end{array}$ & $\begin{array}{c}\text { Expo- } \\
\text { Sure } \\
\text { Time } \\
(\mathbf{m i n})\end{array}$ & $\begin{array}{c}\text { Hexa- } \\
\text { hydrate } \\
\text { Conc } \\
(\mathbf{m o l} / \mathbf{L})\end{array}$ & $\begin{array}{c}\text { Pyrrole } \\
\text { Conc } \\
(\mathbf{m o l} / \mathbf{L})\end{array}$ & $\begin{array}{c}\text { Polymerizatio } \\
\mathbf{n} \\
\text { Time } \\
(\mathbf{h})\end{array}$ & $\begin{array}{c}\text { Outpu } \\
\mathbf{t} \\
\text { Power } \\
(\mathbf{W})\end{array}$ \\
\hline-1 & 5 & 0.1 & 0.05 & 3 & 450 \\
1 & 10 & 0.3 & 0.10 & 24 & 700 \\
\hline
\end{tabular}

After pyrolysis, samples at LTLP and HTHP were subjected to elemental analysis using energy dispersive
X-ray attached to a Hitachi SU3500 scanning electron microscopy.

\section{Results and discussion}

\subsection{Corn cob biochar (CCBc) yield}

Analysis of variance showed that exposure time, output power and their interaction were significant in the $\mathrm{CCBc}$ yield. The corn cob samples pyrolyzed under higher energy denoted by higher exposure time and output power, regardless of other parameter levels, produced a significantly lower amount of biochar compared to those in lower energy level as shown in Fig. 2. Considering these significant parameters, the highest yield of $52.87 \%$ was obtained at low time-low power (LTLP) at $5 \mathrm{~min}$ and $450 \mathrm{~W}$. On the other hand, the lowest yield of $13.99 \%$ was produced at high time-high power (HTHP) of $10 \mathrm{~min}$ and $700 \mathrm{~W}$.

\subsection{Effect of exposure time, output power and their interaction}

Microwave pyrolysis employs thermal decomposition of the biomass leaving primarily carbon in the biochar [15]. As mentioned earlier, there is a general decrease in yield at higher output power (Fig. 2a) and the same trend is also true in the relationship of microwave exposure time and biochar yield (Fig. 2b). This is probably due to the large amount of heat generated within the system at high wattage and longer exposure time. In an atomic-level, this heat comes from the oscillation of molecules as it tries to be in phase with the electromagnetic wave [16]. The movement transforms kinetic energy into dissipated heat which consequently increases the system temperature [17].

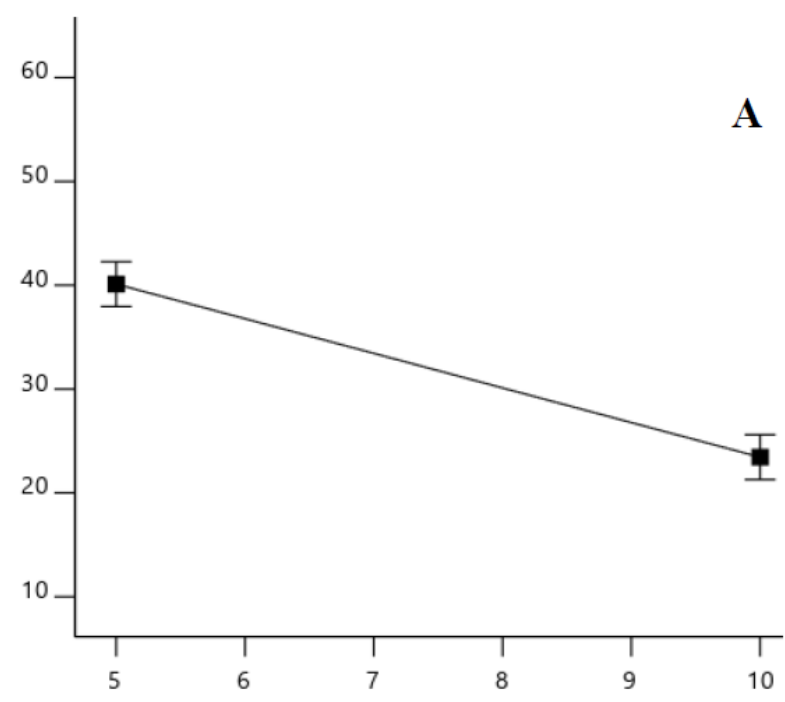

\section{A: Exposure Time (min)}

Figure 2a. Effect of output power on $\mathrm{CCBc}$ yield (one-factor comparison). 


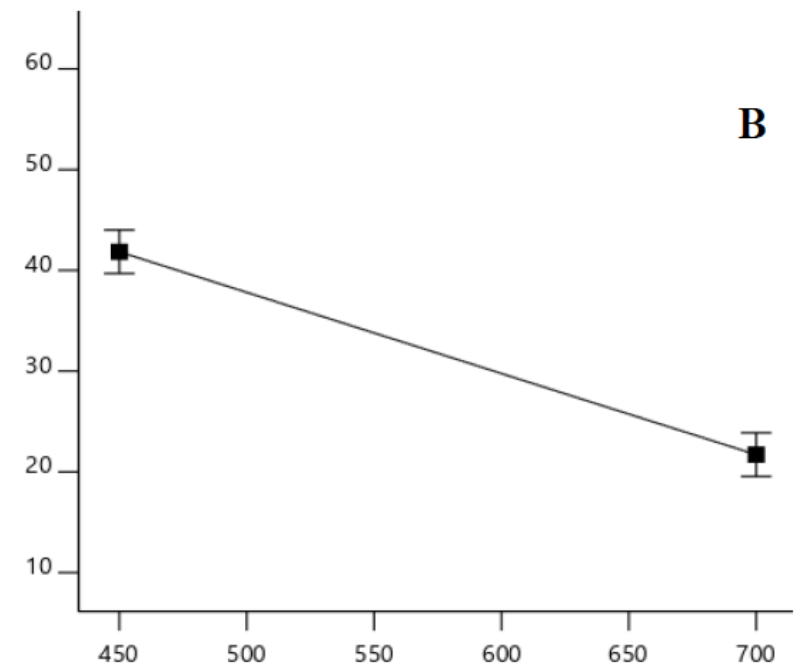

E: Output Power (W)

Figure 2b. Effect of exposure time on $\mathrm{CCBc}$ yield (one-factor comparison).

Due to high temperature effects on the hydrocarbons present in the biomass, it permanently transform into its gaseous form called syngas [18]. Therefore, it is agreeable that higher output power and longer exposure time yields lower biochar product.

On the other hand, Fig. 3 shows the interaction of both factors and its relationship to the CCBc yield. the heating rate experienced at lower power pertains to the slow pyrolysis of corn cob. At this operating condition, lower energy is absorbed per unit time therefore it can be deduced that the maximum temperature attained is lower than that of the system operated at $700 \mathrm{~W}$. Lower temperatures favors char production than syngas [14], hence the significant effect of the interaction.

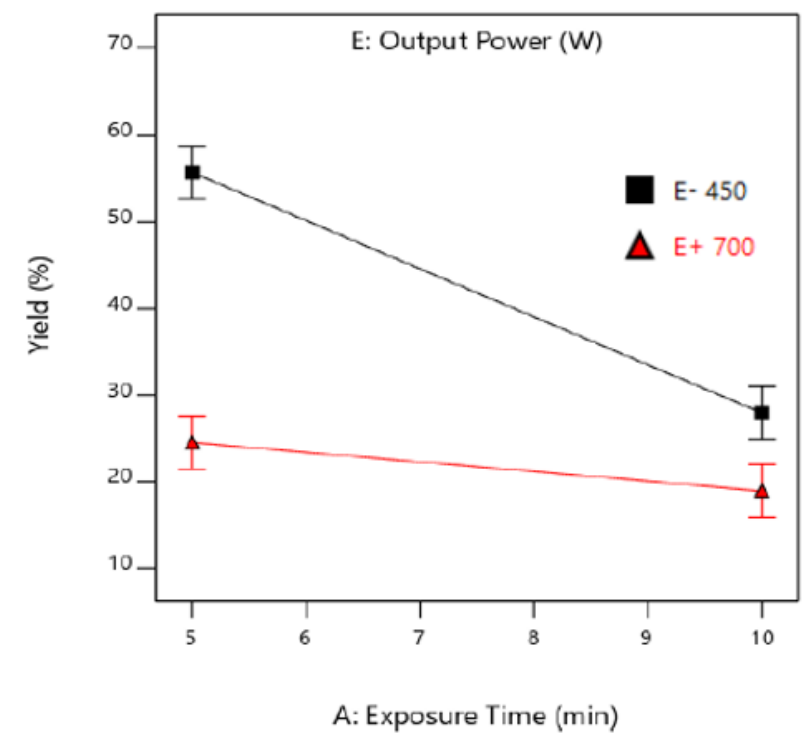

Figure 3. Effect of microwave exposure time and output power interaction on the $\mathrm{CCBc}$ yield.

However, their interaction becomes insignificant, denoted by the overlapping error bars, at high output power of $700 \mathrm{~W}$. At this point, the system experiences high temperatures early in the process as it absorbs high amount of energy per unit time. This environment favors the conversion of many hydrocarbons into syngas rather than thermally decomposing the material [18] as mentioned earlier. At this point, it is suspected that the system approached its ultimate temperature, which was described in the study of Y. F Huang (2008) [19], in less than 5 minutes therefore giving a low biochar yield early in the process and this yield is considered as the fixed carbon content.

\subsection{Effect of pyrrole, catalyst and polymerization time}

From the statistical analysis, varying pyrrole concentration, $\mathrm{FeCl}_{3}$ hexahydrate (catalyst) concentration and polymerization time of polypyrrole has no significant effect on the biochar yield. However, the absence of the following factors will make the pyrolysis impossible since it will only cook the corn cob samples rather than thermally decompose it.

In the experiment, the addition of iron catalyst immediately initiates the in-situ formation of the microwave absorber polypyrrole via wet polymerization of pyrrole monomer into its polypyrrole form, a blackcolored precipitate. Figure 4 shows the color transition in the mixture upon addition of the catalyst. Low concentrations of the reagents were observed to have longer transition times (approximately 2-3 minutes) while high concentrations require shorter times (approximately $<1 \mathrm{~min}$ ). These transitions can be hastened by agitation to properly disperse the reagents in the mixture.

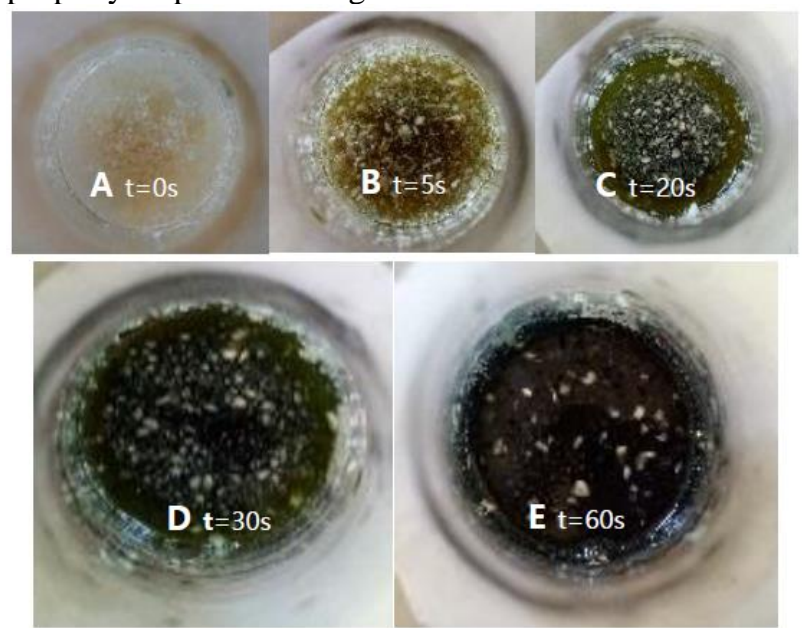

Figure 4. Observed color change (at different time intervals) upon polymerization of pyrrole to polypyrrole upon addition of catalyst solution

The transition time does not guarantee a complete degree of polymerization as observed in the small pieces of light-colored corn cob samples in Figure 4(e). Statistical analysis, however, waives the effect of varying polymerization time on the biochar yield. This probably means that a critical polymerization time, less than the range used, can achieve a polypyrrole solution that produces the same heat generation done in the experimental range [20]. Similarly, the pyrrole 
concentration does not have a significant effect on the thermal decomposition and can also possibly have a critical concentration much lower than the proposed range.

\subsection{Carbon content of biochar}

Raw corncobs (RC) have approximately $42 \%$ carbon After pyrolysis, the carbon content increases at higher temperatures from $55 \%$ [21] up to $92.3 \%$ [22] depending on the operating conditions used in the pyrolysis.

To verify the $\mathrm{CCBc}$ as a viable biochar product, both samples ran at LTLP and HTHP, including both trials, were evaluated for composition analysis through EDX. The percent weight of carbon and oxygen were the major components observed in the biochar. Table 2 shows the average composition analysis of the 2 trials per level.

Table 2. Elemental composition of the biochar using EDX analysis.

\begin{tabular}{ccccc}
\hline PARAMETER & $\mathbf{\% C}$ & $\mathbf{\% O}$ & $\mathbf{\% N}$ & $\mathbf{\% C l}$ \\
\hline $450 \mathrm{~W}, 5 \mathrm{~min}$ & $\mathbf{6 7 . 1 1}$ & 22.45 & 3.69 & 6.73 \\
$($ LTLP) & \pm 1.16 & \pm 0.04 & \pm 0.39 & \pm 0.49 \\
$700 \mathrm{~W}, 10 \mathrm{~min}$ & $\mathbf{8 1 . 3 2}$ & 13.06 & 1.04 & 4.59 \\
$($ HTHP) & \pm 7.59 & \pm 6.24 & \pm 0.32 & \pm 1.04 \\
\hline
\end{tabular}

Results showed that pyrolysis ran at LTLP have more than $20 \%$ increase in average carbon content while HTHP conditions almost doubled the initial carbon content (based on $42 \% \mathrm{C}$ in $\mathrm{RC}$ ). This confirms that the biochar is indeed carbonized under microwave pyrolysis as signified by the increase in elemental carbon. This behavior is contrary to the trend of the biochar yield as discussed previously. However, biochar yield may be decreased but its purity (in terms of \%carbon) is more enhanced. In comparison, lignite has $60-70 \%$ carbon, bituminous coal has $77-87 \%$ carbon and anthracite has over $87 \%$ carbon [23].

The increase in carbon content is due to the continuous liberation of moisture and syngas as the temperature increases inside the microwave. The syngas is mainly composed of $\mathrm{CO}_{2}, \mathrm{CO}, \mathrm{CH}_{4}$ and $\mathrm{H}_{2}$ and can vary in percentage depending on operating conditions. It is the product of tar cracking, char decomposition and reaction between their by-products [24]. Therefore, as the reaction proceeds, the mass decreases due to the escape of the syngas lowering the biochar yield but at the same time saturates most of the carbon in the solid biochar.

Based from the quantitative results, it can be said that, indeed, microwave pyrolysis is successful in carbonizing corn cob wastes into biochar. Moreover, the biochar produced has enough carbon content to become a viable source of carbon-rich materials that is comparable to industrially available carbon-based products such as activated carbon, lignite, bituminous coal, etc. This makes microwave pyrolysis a potential method in producing biochar for fuel-based applications due to its purity in terms of carbon content.

\section{Conclusion}

The significant effect of the exposure time and output power on the $\mathrm{CCBc}$ yield can be majorly attributed to the energy absorbed by the material. At low energy, the system temperature is low enough to promote char production rather than syngas and the degree of carbonization can be modified just by adjusting the exposure time. However, at high output power, the system bypasses carbonization and prefers syngas production due to high temperatures early in the process. Also, there is an observed interaction between the two factors wherein high output power makes the exposure time insignificant due to the high amounts of energy absorbed per unit time early in the process. On the other hand, the other three factors dictate the amount of microwave absorber produced in the system, but their insignificance suggest manipulating the range to obtain optimum values. Lastly, further confirmation of the biochar's carbonization through EDX shows a minimum of $67.11 \%$ or at least $20 \%$ increase in carbon content even at LTLP and has easily double up to $81.32 \%$ at HTHP.

\section{References}

[1] Salman Zafar, "Agricultural Wastes in the Philippines,” Bioenergy consult, 2018. [Online]. Available:

https://www.bioenergyconsult.com/agriculturalresources-in-philippines/. [Accessed: 23-Aug2017].

[2] F. Mushtaq, R. Mat, and F. N. Ani, "A review on microwave assisted pyrolysis of coal and biomass for fuel production," Renew. Sustain. Energy Rev., vol. 39, pp. 555-574, 2014.

[3] N. Bagheri and J. Abedi, "Adsorption of methane on corn cobs based activated carbon," Chem. Eng. Res. Des., vol. 89, no. 10, pp. 20382043, 2011.

[4] Y. Wan et al., "Microwave-assisted pyrolysis of biomass: Catalysts to improve product selectivity, Anal. Appl. Pyrolysis, vol. 86, no. 1, pp. 161-167, 2009 .

[5] K. T. Klasson, "Biochar characterization and a method for estimating biochar quality from proximate analysis results," Biomass and Bioenergy, vol. 96, pp. 50-58, 2017.

[6] Z. Gao, Y. Zhang, N. Song, and X. Li, "Biomassderived renewable carbon materials for electrochemical energy storage," Mater. Res. Lett., vol. 5, no. 2, pp. 69-88, 2017.

[7] M. J. Gronnow et al., "Torrefaction/biochar production by microwave and conventional slow pyrolysis - comparison of energy properties," GCB Bioenergy, vol. 5, no. 2, pp. 144-152, 2013.

[8] C. Ravikumar, P. S. Kumar, S. K. Subhashni, P. $\mathrm{V}$ Tejaswini, and V. Varshini, "Microwave assisted fast pyrolysis of corn cob, corn stover, saw dust and rice straw: Experimental investigation on bio-oil yield and high heating 
values," Sustain. Mater. Technol., vol. 11, pp. 19-27, 2017.

[9] X. Zhao, W. Wang, H. Liu, C. Ma, and Z. Song, "Microwave pyrolysis of wheat straw: Product distribution and generation mechanism," Bioresour. Technol., vol. 158, pp. 278-285, 2014.

[10] P. M. Ingole, A. C. Ranveer, S. M. Deshmukh, and S.K. Deshmukh, "Microwave Assisted Pyrolysis of Biomass: A Review," Int. J. Adv. Technol. Eng. Sci., vol. 4, no. 6, pp. 78-84, 2016.

[11]H. Lei, S. Ren, and J. Julson, "The Effects of Reaction Temperature and Time and Particle Size of Corn Stover on Microwave Pyrolysis," no. 11, pp. 3254-3261, 2009.

[12] Y. P. Huang, C. H. Hou, H. C. Hsi, and J. W. $\mathrm{Wu}$, "Optimization of highly microporous activated carbon preparation from Moso bamboo using central composite design approach," $J$. Taiwan Inst. Chem. Eng., vol. 50, pp. 266-275, 2015.

[13]Z. Januri, S. S. Idris, H. A. Akhawan, N. A. Rahman, S. Matali, and S. F. A. Manaf, "Effect of mass loading and microwave absorber application method on the product from microwave assisted pyrolysis of palm oil mill effluent," Malaysian J. Anal. Sci., vol. 21, no. 2, pp. 470-483, 2017.

[14]C. Wang, D. Ma, and X. Bao, “Transformation of Biomass into Porous Graphitic Carbon Nanostructures by Microwave Irradiation," $J$. Phys. Chem. C, vol. 112, no. 45, pp. 1759617602, 2008.

[15] S. Gadkari, B. Fidalgo, and S. Gu, "Numerical investigation of microwave-assisted pyrolysis of lignin," Fuel Process. Technol., vol. 156, pp. 473-484, 2017.

[16] Arthur von Hippel, "Theory and Applications of RF/Microwave Absorbers," Emerson Cuming Microw. Prod. Inc 28, pp. 1-19, 2012.

[17] M. Miura, H. Kaga, A. Sakurai, T. Kakuchi, and K. Takahashi, "Rapid pyrolysis of wood block by microwave heating," J. Anal. Appl. Pyrolysis, vol. 71, no. 1, pp. 187-199, 2004.

[18] Y. Huang, P. Chiueh, and S. Lo, "Mini review A review on microwave pyrolysis of lignocellulosic biomass," Sustain. Environ. Res., vol. 26, no. 3, pp. 103-109, 2016.

[19] Y. F. Huang, W. H. Kuan, S. L. Lo, and C. F. Lin, "Total recovery of resources and energy from rice straw using microwave-induced pyrolysis," Bioresour. Technol., vol. 99, no. 17, pp. 8252-8258, 2008

[20] E. Hakansson, A. Kaynak, T. Lin, S. Nahavandi, T. Jones, and E. Hu, "Characterization of conducting polymer coated synthetic fabrics for heat generation," Synth. Met., vol. 144, no. 1, pp. 21-28, 2004.

[21]F. Yu, P. H. Steele, and R. Ruan, "Microwave pyrolysis of corn cob and characteristics of the pyrolytic chars," Energy Sources, Part A Recover.
Util. Environ. Eff., vol. 32, no. 5, pp. 475-484, 2010.

[22] F. Zhang, H. Ma, J. Chen, G. D. Li, Y. Zhang, and J. S. Chen, "Preparation and gas storage of high surface area microporous carbon derived from biomass source cornstalks," Bioresour. Technol., vol. 99, no. 11, pp. 4803-4808, 2008.

[23] Hobart King, "Coal," Geology.com. [Online]. Available:https://geology.com/rocks/coal.shtml [Accessed: 06-Apr-2018]

[24] X. Zhao, M. Wang, H. Liu, L. Li, C. Ma, and Z. Song, "A microwave reactor for characterization of pyrolyzed biomass," Bioresour. Technol., vol. 104, pp. 673-678, 2012. 